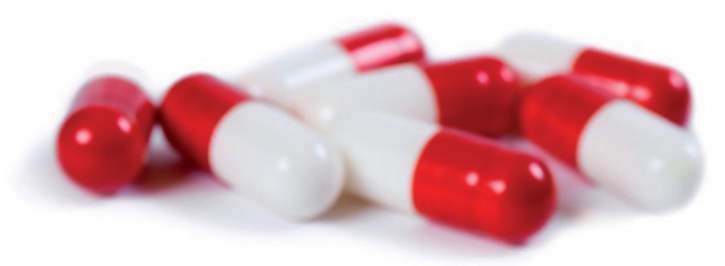

\title{
Ramipril improves walking times in patients
} with PAD

Inhibition of angiotensin-converting enzyme (ACE) with ramipril as an adjunct to standard medical therapy increases both the maximum and the pain-free walking time of patients with peripheral artery disease (PAD). This finding comes from a randomized controlled trial conducted in Australia and published in JAMA. "Given that ramipril is off patent," comments Dr Anna Ahimastos, the lead author on the trial report, "the current findings offer medical professionals worldwide an efficacious and inexpensive agent to dramatically improve clinical symptoms in [patients with] PAD."

PAD affects 27 million individuals in Europe and North America, and claudication (leg muscle pain that is felt when walking, but which is alleviated by rest) occurs in one-third of these patients. However, "research into the development of new therapies to treat $\mathrm{PAD}$ is greatly needed due to the paucity of medical treatments targeting this population," urges Dr William Hiatt from the University of Colorado School of Medicine, CO, USA.

Dr Ahimastos and colleagues noted that "ramipril reduced cardiovascular morbidity and mortality compared to placebo in patients with established atherosclerotic disease, including PAD, in the Heart Outcomes Prevention Evaluation". Therefore, they randomly allocated 212 patients with PAD and intermittent claudication to receive standard medical therapy with the addition of placebo or $10 \mathrm{mg}$ per day of ramipril for 24 weeks.

Ramipril was associated with a $75 \mathrm{~s}$ (95\% CI 60-89 s, $P<0.001)$ increase in the mean pain-free walking time, and a $255 \mathrm{~s}$ (95\% CI 215-295 s, $P<0.001)$ increase in the maximum walking time, compared with standard therapy alone. According to the investigators, this increase in the maximum walking time "corresponds to an average increase in uphill walking distance of $184 \mathrm{~m}$, which would impact appreciably on daily life for these patients".

These improvements are larger than observed with conventional therapy. However, Dr Mary McDermott from Northwestern University Feinberg School of Medicine, IL, USA, writing in an editorial that accompanied the trial report in JAMA, cautions that "the smaller improvement in the placebo group [than in comparable trials] ... contributed to the large relative improvement in the ramipriltreated group".

The researchers also assessed quality of life using the Short-Form 36 Health Survey. Patients taking ramipril had an 8.2 point ( $95 \%$ CI $3.6-11.4$ points, $P=0.02)$ improvement in the physical component of the score, but the mental component of the score was not significantly changed $(0.5$ points, $95 \% \mathrm{CI}$ -0.7 to 1.1 points). Seven patients (6.6\%) taking ramipril withdrew from the trial because of a persistent cough, whereas only two patients $(1.9 \%)$ in the placebo group experienced serious adverse events.
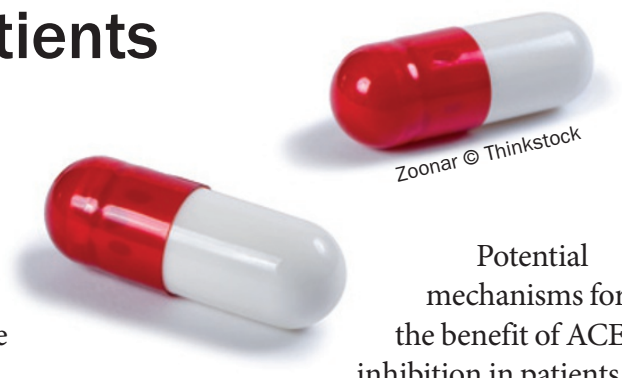

with PAD include increased peripheral blood flow and adaptations in skeletal muscle structure and function. Blood flow might be increased by vasodilatation caused by a reduction in the level of angiotensin II, sympathetic inhibition, and improvement in endothelial function via preservation of bradykinin.

Dr Hiatt suggests that "the implications [of the trial data] may include the use of an ACE inhibitor as an adjuvant to treat claudication, but the primary and approved use of these drugs is to ... treat hypertension. Therefore, for many patients, an ACE inhibitor may already be part of their overall medical management."

"Currently, using ramipril to treat claudication would be 'off-label' and, therefore, physicians should use caution in this setting," warns Dr Hiatt. To address this shortcoming, Dr Ahimastos and colleagues now plan "to build a case for expansion of the regulatory guidelines to include claudication as an indication for ramipril".

Gregory B. Lim

Original article Ahimastos, A. A. et al. Effect of ramipril on walking times and quality of life among patients with peripheral artery disease and intermittent claudication: a randomized controlled trial. JAMA 309, 453-460 (2013) 